\title{
Simultaneous Wireless Information and Power Transfer Mechanism in Interference Alignment Relay Networks
}

\author{
Fahui Wu, ${ }^{1}$ Lin Xiao, ${ }^{2}$ Dingcheng Yang, ${ }^{2}$ Laurie Cuthbert, ${ }^{3}$ and Xiaoping Liu' \\ ${ }^{1}$ Jiangxi Province Key Laboratory of Intelligent Information Systems, Nanchang University, Nanchang, China \\ ${ }^{2}$ Information Engineering School, Nanchang University, Nanchang, China \\ ${ }^{3}$ Queen Mary University of London, London, UK \\ Correspondence should be addressed to Lin Xiao; xiaolin@ncu.edu.cn
}

Received 5 July 2016; Revised 20 September 2016; Accepted 29 September 2016

Academic Editor: Floriano Scioscia

Copyright (C) 2016 Fahui Wu et al. This is an open access article distributed under the Creative Commons Attribution License, which permits unrestricted use, distribution, and reproduction in any medium, provided the original work is properly cited.

\begin{abstract}
This paper considers a simultaneous wireless information and power transfer (SWIPT) mechanism in an interference alignment (IA) relay system, in which source nodes send wireless information and energy simultaneously to relay nodes, and relay nodes forward the received signal to destination nodes powered by harvested energy. To manage interference and utilize interference as energy source, two-SWIPT receiver is designed, namely, power splitting (PS), and antennas switching (AS) has been considered for relay system. The performance of AS- and PS-based IA relay systems is considered, as is a new energy cooperation (ECop) scheme that is proposed to improve system performance. Numerical results are provided to evaluate the performance of all schemes and it is shown from the simulations that the performance of proposed ECop outperformed both AS and PS.
\end{abstract}

\section{Introduction}

Recently, energy harvesting (EH) from radio-frequency (RF) signals has drawn significant attention as it is a promising solution to prolong the lifetime of energy constrained wireless networks, for example, sensor based wireless networks. Since it has practical valuable for enabling both wireless data and wireless energy transmission, $\mathrm{EH}$ has a promising future for green communications. Simultaneous wireless information and power transfer (SWIPT) was first proposed in [1], where SWIPT in a point-to-point single-antenna additive white Gauss noise (AWGN) channel was studied from an information-theoretic standpoint. In [2], Grover extended [1] to frequency-selective single-antenna AWGN channels, where nontrivial tradeoff between information rate and harvested energy was demonstrated by varying power allocation over frequency. Optimal designs that take into account cochannel interference of wireless information and power transfer were studied in [3] to achieve different outage energy tradeoff as well as rate-energy tradeoff.

Since the application of multiple-input multiple-output (MIMO) technology could significantly enhance the transfer efficiency of both energy and information, SWIPT for MIMO channels has been investigated in [4-6]. In [4], Zhang and Ho studied the performance limits of a three-node MIMO. The work in [4] was extended to cases with imperfect channel state information (CSI) at the transmitter in [5]. In [6], when the CSI is not available at the transmitter, a transmitter design based on random beamforming is proposed for a multiple-input single-output (MISO) SWIPT system with artificial channel fading generated over quasi-static channels. SWIPT is studied in orthogonal frequency division multiplexing (OFDM) systems in [7-9], in broadcast channels [10], and in interference channels [11, 12]. In addition, [13] studied SWIPT in MIMO-OFDMA systems and proposed a SWIPT-enabled architecture to study the power control problem in broadband wireless systems. It is well known that MIMO relay networks can markedly improve spectrum utilization and simultaneously enhance link reliability [14, 15], as well as being an efficient way to provide high quality service at low cost [14-16]. More importantly, the concept of SWIPT is more applicable for short-distance applications since wireless power transfer (WPT) will suffer from high attenuation due to path-loss. MIMO relay systems involving 


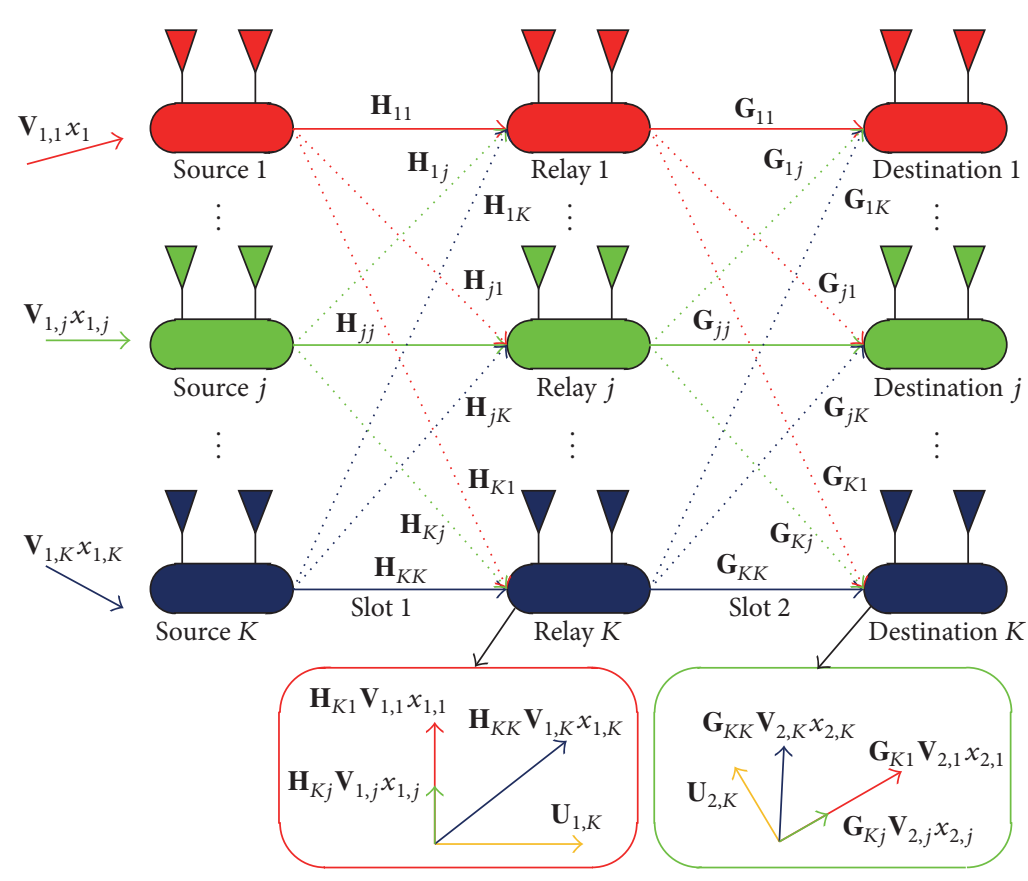

Figure 1: System model of $K$-user interference relay channel.

an energy harvesting receiver were studied in [17], in which the joint optimal source and relay precode were designed to achieve different tradeoffs between the energy transfer and the information rate. In [18] a relaying protocol based on time switching (TS) and power splitting (PS) is proposed to enable energy harvesting and information processing at the relay. In [19], the throughput in a classic three-node Gaussian relay channel with decode-and-forward (DF) relaying is analyzed.

Interference is well known as being one of the major challenges in the transceiver design of wireless communications. A promising solution to interference management in wireless networks is interference alignment (IA) that has proved its optimality to approach the Shannon capacity of interference networks at high signal-to-noise ratio (SNR) [20]. In IA, the transmitted signals of all the users are jointly designed to constrain all the interference into certain subspaces at the unintended receivers, and the desired signal can be obtained from the remaining interference-subspaces by the decoding matrices at each receiver. To obtain the coding and decoding matrices, numerical iteration is proposed in [20], and the feasibility conditions of IA in multiuser MIMO networks were studied in [21]. Due to its outstanding performance, IA has been successfully applied in various networks [22].

The study of SWIPT in IA networks is still at an early stage: although some fundamental work has been done [23, 24], there is not much reported in the literature. In this paper, SWIPT in IA relay networks has been studied, with the performance of antennas switching (AS) and PS-based SWIPT being analyzed.

The system sum-rate maximum problem is formulated as a three-step optimization problem. The problem is transformed to a convex problem that can be solved easily. Combining the transfer characteristic of AS and PS, an energy cooperation scheme is proposed that can achieve better performance than either the AS or PS scheme. Also, the degree of freedom (DoF) condition is more flexible than in the AS scheme.

SWIPT has proved to be more efficient in spectrum usage than transmitting information and energy in orthogonal time or frequency channels [1]. To settle the interference issue in communication systems, existing interference management techniques, for example, interference cancellation, attempt to avoid or mitigate interference through spectrum scheduling. However, with RF energy harvesting, harmful interference can be turned into useful energy through a scheduling policy. This paper provides new vision to mitigate interference and facilitate SWIPT. Furthermore, cooperative relaying techniques can extend the coverage of source node and help to overcome fading and attenuation by using intermediate relay nodes, resulting in improved network performance in terms of efficiency and reliability. Our paper presents tentative exploration in interference management and improves energy efficiency in relay network; our results can be applied to next generation communication system with ultra-densification network infrastructure.

This paper is organized as follows: Section 2 introduces the system model and problem formulations; Section 3 presents the solutions for the formulated problems; Section 4 provides numerical examples to validate our results and compare the performances; and Section 5 concludes the paper.

\section{System Model}

As shown in Figure $1, K$-user interference relay channel is considered, that is, $K$ transmission links of transmitter-relayreceiver. 
It is assumed that $M_{k}, N_{k}$, and $T_{k}$ antennas are fitted at $k$ th source node, relay node, and destination node, respectively; $d_{k}^{S}$ and $d_{k}^{R}$ data streams are transmitted by $k$ th source node and relay node. We assume there is no direct link between the source and the destination node so that an intermediate DF relay assists the transmission of the source messages to the destination. The energy harvesting enabled relay nodes to first harvest energy and receive information simultaneously through RF signals from the source nodes. Next, the relay nodes use the harvested energy as a source of transmission power to forward the decoded source information to the destination nodes. It is assumed that the processing power required by the information decoding circuitry at the relay is negligible as compared to the power used for signal transmission from the relay to the destination.

Communication is performed in two orthogonal time slots and it is assumed that perfect channel state information (CSI) of the network is available at all the nodes. In this paper, to avoid interference between users, linear IA in the spatial dimension is considered, that is, IA in multiuser MIMO systems. This paper mainly concentrates on the information and power transfer in the IA networks instead of degrees of freedom, so we assume that the streams for each node are the same; that is, $d_{k}^{S}=d_{k}^{S}=d, \forall k=1,2, \ldots, K$. Moreover, symmetric networks are considered, and thus all the nodes have the same parameters; that is, $M_{k}=M, N_{k}=N$, and $T_{k}=T, \forall k=1,2, \ldots, K$.

Consider Slot 2 firstly that is the conventional IA network with only information transmission being performed. The received signal at destination node $k$ can be represented as

$$
\begin{aligned}
y_{k}^{D}= & \mathbf{U}_{2, k}^{H} \mathbf{G}_{k k} \mathbf{V}_{2, k} x_{2, k}+\sum_{i=1, i \neq k}^{K} \mathbf{U}_{2, k}^{H} \mathbf{G}_{k i} \mathbf{V}_{2, i} x_{2, i} \\
& +\mathbf{U}_{2, k}^{H} n_{2, k} .
\end{aligned}
$$

$x_{2, k}$ is $d_{k}^{R} \times 1$ data symbol transmitted by the relay node. The average power constraint is denoted as $\operatorname{Tr}\left(\mathbb{E}\left\{x_{2, k} x_{2, k}^{H}\right\}\right)=$ $\operatorname{Tr}\left(\mathbf{S}_{2, k}\right)=P_{2, k}$, where $\mathbf{S}_{2, k}$ denotes the covariance matrix of $x_{2, k} . \mathbf{G}_{k i} \in \mathbb{C}^{N \times T}$ is the channel gain matrix from relay node $i$ to destination node $k$.

For the arbitrary $k$ th relay node, its signal $x_{2, k}$ is precoded by a precoding matrix $\mathbf{V}_{2, k} \in \mathbb{C}^{T \times d_{k}^{R}}$. The coded signal $\mathbf{V}_{2, k} x_{2, k}$ is transmitted by the antennas from relay node $k$ to destination node $k$, which will also cause interference at other unintended destination nodes. At $k$ th destination node, the interferences from other relay nodes, $\mathbf{G}_{k j} \mathbf{V}_{2, j} x_{2, j}, \forall j \neq k$ are constrained into the same subspace that is orthogonal to the unitary interference suppression matrix $\mathbf{U}_{2, k} \in \mathbb{C}^{N \times d_{k}^{R}}$, through the cooperation of the precoding matrices of the other users. Thus, the desired signal of $k$ th destination node can be recovered as $\mathbf{U}_{2, k}^{H} \mathbf{G}_{k k} \mathbf{V}_{2, k} x_{2, k}$ at $k$ th destination node. $n_{2, k} \in \mathbb{C}^{N \times 1}$ represents the additive white Gaussian noise vector at destination node $k$ with distribution $\mathscr{C} \mathscr{N}\left(0, \sigma^{2}\right)$.

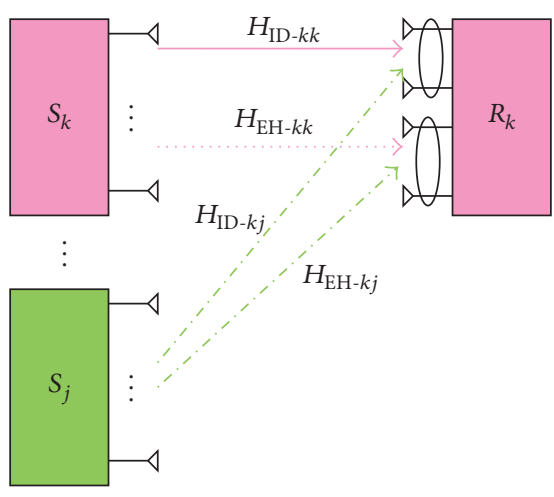

$$
\begin{aligned}
& \longrightarrow \text { Information } \\
& \ldots \rightarrow>\text { Energy } \\
& \cdot-\rightarrow \text { Interference }
\end{aligned}
$$

Figure 2: Antennas switching model.

When IA is feasible, the interferences among users can be assumed to be perfectly eliminated if the following conditions are satisfied $[20,21]$ :

$$
\begin{aligned}
\mathbf{U}_{2, k} \mathbf{G}_{k j} \mathbf{V}_{2, j} & =0, \quad \forall j \neq k, \\
\operatorname{rank}\left(\mathbf{U}_{2, k} \mathbf{G}_{k k} \mathbf{V}_{2, k}\right) & =d_{k}^{R} .
\end{aligned}
$$

The feasible condition for IA can be given as

$$
M+N \geq(K+1) d .
$$

Then (1) can be simplified as

$$
y_{k}^{D}=\mathbf{U}_{2, k}^{H} \mathbf{G}_{k k} \mathbf{V}_{2, k} x_{2, k}+\mathbf{U}_{2, k}^{H} n_{2, k} .
$$

The rate of $k$ th destination node can be described as

$$
R_{2, k}=\log \left|\mathbf{I}+\mathbf{U}_{2, k}^{H} \mathbf{G}_{k k} \mathbf{V}_{2, k} \mathbf{S}_{2, k} \mathbf{V}_{2, k}^{H} \mathbf{G}_{k k}^{H} \mathbf{U}_{2, k}\right|,
$$

where $\operatorname{Tr}\left(\mathbf{S}_{2, k}\right)=P_{2, k} \leq Q$ and $Q$ denotes the harvested energy in Slot 1.

Next, consider the joint information and power transfer in Slot 1 . Assume that each relay node can decode information and harvest energy at the same time. The source node transfers energy and information to the relay node through two mechanisms, AS and PS.

In the AS scheme, receiving antennas are divided into two groups with one group switched to information decoding and the other group to energy harvesting as shown in Figure 2.

We assume that each relay node switches $L$ antennas from all $N$ antennas for information decoding (ID), and the remaining $N-L$ antennas are used for $\mathrm{EH}$. The number of ID antennas should satisfy the feasible condition; that is,

$$
L+M \geq(K+1) d .
$$

The channel matrix between source node $j$ and the ID antennas of relay node $k$ is denoted as $H_{\mathrm{ID}-k j} \in \mathbb{C}^{L \times M}$, which is composed by $L$ rows of $H_{k j}$ and adopted to obtain 


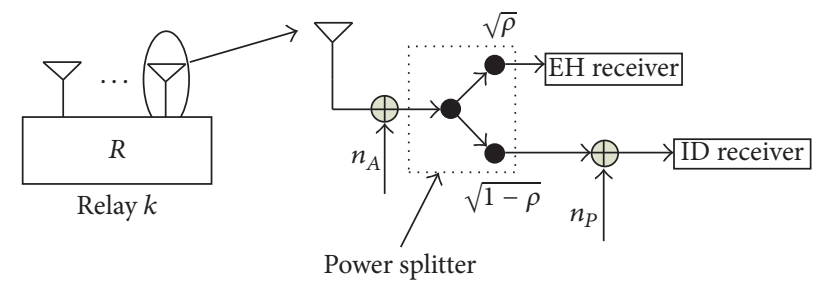

FIGURE 3: Power splitting model.

the coding matrices. Assume that the interference can be eliminated completely, and the rate of the $k$ th relay noted can be described as

$$
R_{1, \mathrm{AS}-k}=\log _{2}\left|\mathbf{I}_{d_{k}}+\mathbf{U}_{1, k}^{H} \mathbf{H}_{\mathrm{ID}-k k} \mathbf{V}_{1, k} \mathbf{S}_{k} \mathbf{V}_{1, k}^{H} \mathbf{H}_{\mathrm{ID}-k k}^{H} \mathbf{U}_{1, k}\right| .
$$

For power transfer, the channel matrix between source node $j$ and the relay node $k$ is $\mathbf{H}_{\mathrm{EH}-k j} \in \mathbb{C}^{(N-L) \times M}$. The harvested energy at relay node $k$ can be described as

$$
Q_{\mathrm{AS}-k}=\zeta \operatorname{Tr}\left(\sum_{i=1}^{K} \mathbf{H}_{\mathrm{EH}-k i} \mathbf{V}_{i} \mathbf{S}_{i} \mathbf{V}_{i}^{H} \mathbf{H}_{\mathrm{EH}-k i}^{H}\right)
$$

where $\zeta(0<\zeta<1)$ is the energy conversion efficiency at the relay node and the background noise at the $\mathrm{EH}$ receiver is negligible and thus can be ignored.

For the PS scheme, the received signal at each antenna is split into two separate signal streams with different power levels, one sent to the $\mathrm{EH}$ receiver and the other to the ID receiver, as shown in Figure 3.

The received signal from the antenna is first corrupted by a Gaussian noise denoted by $n_{A} \sim \mathscr{C} \mathcal{N}\left(0, \sigma_{A}^{2}\right)$ in the RF-band. The RF-band signal is then fed into a power splitter, which is assumed to be perfect without any noise induced. The signal split to the ID receiver then goes through a sequence of standard operations to be converted from the RF band to baseband. During this process, the signal is additionally corrupted by another noise $n_{P} \sim \mathscr{C} \mathscr{N}\left(0, \sigma_{P}^{2}\right)$, which is independent of $n_{A}$.

For $j$ th antenna at $k$ th relay node, the portion of power for EH is denoted as $\rho_{k j}, 0 \leq \rho_{k j} \leq 1$ and that for ID is $1-\rho_{k j}$, $1 \leq j \leq N$. Then the rate of $k$ th relay node can be calculated as

$$
\begin{aligned}
& R_{1, \mathrm{PS}-k}=\log \mid \mathbf{I} \\
& \quad+\mathbf{U}_{1, k}^{H} \boldsymbol{\Lambda}_{k}^{1 / 2} \boldsymbol{\Psi}_{k 1} \overline{\boldsymbol{\Lambda}}_{k}^{1 / 2} \mathbf{U}_{1, k}^{H}\left(\mathbf{U}_{k}^{H}\left(\overline{\boldsymbol{\Lambda}}_{k}^{1 / 2}\left(\sigma_{A}^{2} \mathbf{I}+\boldsymbol{\Psi}_{k 2}\right) \overline{\boldsymbol{\Lambda}}_{k}^{1 / 2}+\sigma_{P}^{2}\right)\right. \\
& \left.\quad \cdot \mathbf{U}_{k}\right)^{-1} \mid
\end{aligned}
$$

where

$$
\begin{aligned}
& \boldsymbol{\Psi}_{k 1}=\mathbf{H}_{k k} \mathbf{V}_{1, k} \mathbf{S}_{1, k} \mathbf{V}_{1, k}^{H} \mathbf{H}_{k k}^{H}, \\
& \Psi_{k 2}=\sum_{i=1, i \neq k}^{K} \mathbf{H}_{k i} \mathbf{V}_{i} \mathbf{S}_{i} \mathbf{V}_{i}^{H} \mathbf{H}_{k i}^{H},
\end{aligned}
$$

where $\boldsymbol{\Lambda}_{k}=\operatorname{diag}\left(\rho_{k 1}, \rho_{k 2}, \ldots, \rho_{k N}\right)$ and $\overline{\boldsymbol{\Lambda}}_{k}=\mathbf{I}-\boldsymbol{\Lambda}_{k}$ represents the partition of power for all $N$ receiving antennas at $k$ th relay node. The harvested energy at $k$ th relay node can be calculated similar to (8) as

$$
Q_{\mathrm{PS}-k}=\zeta \operatorname{Tr}\left(\sum_{i=1}^{K} \boldsymbol{\Lambda}_{k} \mathbf{H}_{k i} \mathbf{V}_{1, i} \mathbf{S}_{1, i} \mathbf{V}_{1, i}^{H} \mathbf{H}_{k i}^{H}\right) .
$$

For the relay system, we assume Slot $1=$ Slot 2 , so the system rate of $k$ th relay transmission link can be described as

$$
R_{k}=\frac{1}{2} \min \left(R_{1, k}, R_{2, k}\right) .
$$

\section{Problem Formulation}

3.1. Antenna Switching-Based Relaying. In AS, it supposed that $L$ antennas are selected from all $N$ for ID; hence, there are $a=\left(C_{N}^{L}\right)^{K}$ feasible combinations totally. The optimal AS scheme can be found through searching the optimal one from all feasible antennas combinations. Assume that the global channel knowledge is available to all users. For a selected AS scheme, the optimization problem can be described as follows:

$$
\begin{aligned}
\max _{\mathbf{S}_{1,1}, \ldots, \mathbf{S}_{1, K}, \mathbf{S}_{2,1}, \ldots, \mathbf{S}_{2, K}} & \sum_{k=1}^{K} \min \left(R_{1, k}, R_{2, k}\right) \\
\text { s.t. } \quad & \operatorname{Tr}\left(\mathbf{S}_{1, k}\right) \leq P, \quad k=1, \ldots, K \\
& \operatorname{Tr}\left(\mathbf{S}_{2, k}\right) \leq Q_{\text {AS- } k}, \quad k=1, \ldots, K \\
& \mathbf{S}_{1, k} \geq \mathbf{0}, \\
& \mathbf{S}_{2, k} \geq \mathbf{0},
\end{aligned}
$$

$$
k=1, \ldots, K
$$

Theorem 1. The power allocation problem (P1) can be divided into $K$ independent subproblems for $K$ links.

Proof. Since the transmission in Slot 2 is powered by the harvested energy in Slot 1, we have $P_{2, k}=Q_{\mathrm{AS}-k}$. Moreover, consider perfect IA and optimal power allocation on Slot 2; the rate of $k$ th link in Slot 2 is independent of any other link. The two-hop optimization problem can be regarded as a rateenergy tradeoff problem in Slot 1:

$$
\begin{aligned}
\max _{\mathbf{S}_{1,1}, \ldots, \mathbf{S}_{1, K}} & \sum_{k=1}^{K}\left(\alpha_{k} R_{1, k}+\left(1-\alpha_{k}\right) Q_{\mathrm{AS}-k}\right) \\
\text { s.t. } & \operatorname{Tr}\left(\mathbf{S}_{1, k}\right) \leq P \\
& \mathbf{S}_{1, k} \geq \mathbf{0}, \quad k=1, \ldots, K,
\end{aligned}
$$

where $0 \leq \alpha_{k} \leq 1$ denotes the weight allocated to the rate at $k$ th relay, to make a tradeoff between EH and IT. For the AS combination determined, to achieve a maximum sum rate of $K$ relay links, the value of $\alpha_{k}$ should be proper setting. 
For a given $\alpha_{k}$, the power allocation problem (P1-a) can be divided into $K$ independent subproblems for $K$ links:

$$
\begin{aligned}
\sum_{k=1}^{K} & \max \left(\alpha_{k} R_{\mathrm{AS}-1, k}+\left(1-\alpha_{k}\right) Q_{\mathrm{AS}-k}\right) \\
& =\sum_{k=1}^{K} \max \left(\alpha_{k} R_{\mathrm{AS}-1, k}\right. \\
& \left.+\left(1-\alpha_{k}\right) \zeta \operatorname{Tr}\left(\sum_{i=1}^{K} \mathbf{H}_{\mathrm{EH}-k i} \mathbf{V}_{i} \mathbf{S}_{i} \mathbf{V}_{i}^{H} \mathbf{H}_{\mathrm{EH}-k i}^{H}\right)\right) \\
& =\sum_{k=1}^{K} \max \left(\alpha_{k} R_{\mathrm{AS}-1, k}\right. \\
& \left.+\operatorname{Tr}\left(\sum_{i=1}^{K}\left(1-\alpha_{i}\right) \zeta \mathbf{H}_{\mathrm{EH}-i k} \mathbf{V}_{k} \mathbf{S}_{k} \mathbf{V}_{k}^{H} \mathbf{H}_{\mathrm{EH}-i k}^{H}\right)\right) \\
& =\sum_{k=1}^{K} \max \left(\alpha_{k} R_{\mathrm{AS}-1, k}+E_{\mathrm{AS}-k}\right) .
\end{aligned}
$$

The decision variables are $\mathbf{S}_{1, k}, k=1, \ldots, K$. Thus, the optimization problem can be rewritten as the following $K$ parallel subproblems as

$$
\begin{aligned}
\max _{\mathbf{S}_{1, k}} & \max \alpha_{k} R_{1, k}+\left(1-\alpha_{k}\right) Q_{\mathrm{AS}-k} \\
\text { s.t. } & \operatorname{Tr}\left(\mathbf{S}_{1, k}\right) \leq P, \\
& \mathbf{S}_{1, k}^{S} \geq 0,
\end{aligned}
$$

where $k=1, \ldots, K$.

According to Theorem 1, it is observed that, for a given antenna combination and weight value combination, each source node can determine its own power allocation simultaneously to achieve global optimization. Consider $k$ th source node; the power allocation is denoted as (13) which is convex and can be solved effectively by the interior point method [25].

3.2. Power Splitting-Based Relaying. Consider the PS-based IA relay systems; to eliminate interference completely, uniform power splitting (UPS) should be used [24]; that is, $\rho_{k 1}=$ $\rho_{k 2}=\cdots=\rho_{k T}$.

It is assumed first that each source node has only one data stream; that is, $d=1$. The optimal power splitting ratio at $k$ th relay node is

$$
\begin{aligned}
& \rho_{k}^{*} \\
& =\frac{\zeta \operatorname{Tr}\left(\sum_{i=1}^{K} \mathbf{H}_{k i} \mathbf{V}_{1, i} \mathbf{V}_{1, i}^{H} \mathbf{H}_{k i}^{H}\right)}{\zeta \operatorname{Tr}\left(\sum_{i=1}^{K} \mathbf{H}_{k i} \mathbf{V}_{1, i} \mathbf{V}_{1, i}^{H} \mathbf{H}_{k i}^{H}\right)+\mathbf{U}_{2, k}^{H} \mathbf{G}_{k k} \mathbf{V}_{2, k} \mathbf{V}_{2, k}^{H} \mathbf{G}_{k k}^{H} \mathbf{U}_{2, k}} .
\end{aligned}
$$

Proof. $d=1$ : the rate of $k$ th user in Slot 1 is

$$
\begin{aligned}
R_{1, k} & =\left|\mathbf{I}+\mathbf{U}_{1, k}^{H} \bar{\Lambda}_{k}^{1 / 2} \mathbf{H}_{k k} \mathbf{V}_{1, k} \mathbf{S}_{1, k} \mathbf{V}_{1, k}^{H} \mathbf{H}_{k k}^{H} \bar{\Lambda}_{k}^{1 / 2} \mathbf{U}_{1, k}^{H}\right| \\
& =\left|\mathbf{I}+P_{1, k}\left(1-\rho_{k}\right) \mathbf{U}_{1, k}^{H} \mathbf{H}_{k k} \mathbf{V}_{1, k} \mathbf{V}_{1, k}^{H} \mathbf{H}_{k k}^{H} \mathbf{U}_{1, k}^{H}\right| .
\end{aligned}
$$

Corresponding harvested energy in Slot 1 is

$$
\begin{aligned}
Q_{\text {PS }-k} & =\zeta \operatorname{Tr}\left(\sum_{i=1}^{K} \boldsymbol{\Lambda}_{k} \mathbf{H}_{k i} \mathbf{V}_{1, i} \mathbf{S}_{1, i} \mathbf{V}_{1, i}^{H} \mathbf{H}_{k i}^{H}\right) \\
& =P_{1, k} \rho_{k} \zeta \operatorname{Tr}\left(\sum_{i=1}^{K} \mathbf{H}_{k i} \mathbf{V}_{1, i} \mathbf{V}_{1, i}^{H} \mathbf{H}_{k i}^{H}\right) .
\end{aligned}
$$

The rate of $k$ th user in Slot 2 is

$$
\begin{aligned}
R_{2, k} & =\log \left|\mathbf{I}+\mathbf{U}_{2, k}^{H} \mathbf{G}_{k k} \mathbf{V}_{2, k} \mathbf{S}_{2, k} \mathbf{V}_{2, k}^{H} \mathbf{G}_{k k}^{H} \mathbf{U}_{2, k}^{H}\right| \\
& =\log \left|\mathbf{I}+Q_{\text {PS- } k} \mathbf{U}_{2, k}^{H} \mathbf{G}_{k k} \mathbf{V}_{2, k} \mathbf{V}_{2, k}^{H} \mathbf{G}_{k k}^{H} \mathbf{U}_{2, k}^{H}\right| .
\end{aligned}
$$

Let $R_{1, k}=R_{2, k}$; we have

$$
\begin{gathered}
P_{1, k}\left(1-\rho_{k}\right) \mathbf{U}_{1, k}^{H} \mathbf{H}_{k k} \mathbf{V}_{1, k} \mathbf{V}_{1, k}^{H} \mathbf{H}_{k k}^{H} \mathbf{U}_{1, k}^{H} \\
=Q_{\mathrm{PS}-k} \mathbf{U}_{2, k}^{H} \mathbf{G}_{k k} \mathbf{V}_{2, k} \mathbf{V}_{2, k}^{H} \mathbf{G}_{k k}^{H} \mathbf{U}_{2, k}^{H} .
\end{gathered}
$$

Substituting (16) into (18), the optimal power splitting ratio can be calculated and denoted as (14).

When $d>1$, the power allocation at each source node, PS ratio, and weight value at each relay node should be jointly optimized. For a give rate weight value, the optimization problem can be formulated as

$$
\begin{aligned}
\max _{\mathbf{S}_{1,1}, \ldots, \mathbf{S}_{1, K}, \boldsymbol{\Lambda}_{1}, \ldots, \boldsymbol{\Lambda}_{K}} & \sum_{k=1}^{K} \alpha_{k} R_{1, k}+\left(1-\alpha_{k}\right) Q_{\mathrm{PS}-k} \\
\text { s.t. } \quad & 0 \leq \rho_{k} \leq 1, \quad k=1, \ldots, K \\
& \operatorname{Tr}\left(\mathbf{S}_{1, k}\right) \leq P, \\
& \mathbf{S}_{1, k} \geq 0,
\end{aligned}
$$

$$
k=1, \ldots, K
$$

In (P2), if $\rho_{k}$ are given, (P2) can be divided into $K$ independent subproblems for $K$ links as for (P1). The optimal solution for PA $S_{k}^{S^{*}}$ can be obtained through applying the interpoint method for the UPS scheme.

3.3. Energy Cooperation. Since the AS scheme has better rate performance and PS scheme has advantages on the harvested energy [24], we consider an energy cooperation (ECop) scheme where the PS relays share harvested energy with AS relays.

It is assumed that only $K$ th relay node adopts AS scheme and the reminder relay nodes are PS schemes. The IA relay systems are still symmetric networks where all the nodes have the same parameters; that is, $M_{k}=M, T_{k}=T, N_{k}=N$, and $d_{k}=d, \forall k=1,2, \ldots, K$.

Prior to describing the optimization problem, we first check the feasibility condition of DoF. Assume that the $L$ antennas are selected for ID at $K$ th relay node, the symmetric networks become asymmetric systems, and the feasibility condition should be reconsidered. From [26], the 
TABLE 1: Comparison of highest DoF for different schemes.

\begin{tabular}{lccc}
\hline$(M, N, L, K)$ & $\begin{array}{c}\text { PS: proper } d \\
\text { given by (2) (3) }\end{array}$ & $\begin{array}{c}\text { AS: proper } d \\
\text { given by (6) }\end{array}$ & $\begin{array}{c}\text { ECop: proper } d \\
\text { given by (20a) } \\
\text { and (20b) }\end{array}$ \\
\hline$(6,6,5,3)$ & 3 & 2 & 2 \\
$(5,5,2,3)$ & 2 & 1 & 2 \\
$(6,4,3,3)$ & 2 & 2 & 2 \\
$(4,4,3,3)$ & 2 & 1 & 1 \\
$(6,6,2,4)$ & 2 & 1 & 2 \\
$(6,3,2,4)$ & 1 & 1 & 1 \\
\hline
\end{tabular}

total number of equations and the total number of variables are given by

$$
\begin{aligned}
N_{v} & =\sum_{i=1}^{K-1} d_{i}\left(M_{i}+N_{i}-2 d_{i}\right)+d_{K}\left(M_{K}+L-2 d_{K}\right) \\
& =[K(M+N-2 d)+(L-N)] d, \\
N_{e} & =\sum_{i=1}^{K} \sum_{j=1, j \neq i}^{K} d_{i} d_{j} .
\end{aligned}
$$

The IA condition is proper if $N_{v} \geq N_{e}$; that is,

$$
\frac{(M+N)}{K+1}-\frac{(N-L)}{K(K+1)} \geq d .
$$

Moreover, the ID receiver can simultaneously decode up to $L$ streams, which is

$$
L \geq d
$$

Note that, in the energy cooperation IA, the feasibility condition is stricter than condition (6). Table 1 compares the highest achievable DoF for given $(M, N, L, K)$.

It is observed that energy cooperation has a better DoF than AS IA and the energy cooperation network will always have a lower DoF than PS, since Kth AS relay node prevents the network being symmetric anymore.

Now consider the design problem for the ECoP IA; our aim is to maximize the sum-rate of all relay links subject to individual rate constraints at PS links, given by $C_{k}, k=$ $1, \ldots, K-1$.

Remark 2. For $k$ th $(k=1, \ldots, K-1)$ PS relay link, if

$$
\begin{array}{r}
\left|\mathbf{U}_{1, k}^{H} \mathbf{H}_{k k} \mathbf{V}_{1, k}\right|^{2}>\left|\mathbf{U}_{1, K}^{H} \mathbf{H}_{K K} \mathbf{V}_{K K}\right|^{2}, \\
k=1, \ldots, K-1, \\
\left|\mathbf{U}_{2, k}^{H} \mathbf{G}_{k k} \mathbf{V}_{2, k}\right|^{2}>\left|\mathbf{U}_{2, K}^{H} \mathbf{G}_{K K} \mathbf{V}_{2, K}\right|^{2}, \\
k=1, \ldots, K-1 .
\end{array}
$$

Specifically, if $k$ th $(k=1, \ldots, K-1)$ relay link has a better rate performance than $K$ th link in both slots, $k$ th relay node would not share its harvested energy with an AS relay node. The design objective of $k$ th $(k=1, \ldots, K-1)$ relay links is still energy-rate tradeoff.
Now the energy cooperation scheme is described as follows.

First, calculate the energy requirement to maximize the system rate of the AS link. More precisely, find an antenna combination that achieves the maximum rate in Slot 1 and then calculate the energy requirement gap at relay nodes for forwarding the received information.

Next, consider how much energy should be shared for the ECop available relay node. We define a parameter called energy-rate ratio (ERR) to compare the instantaneous $\mathrm{EH}$ capacity to ID capability of a relay node. The ERR of relay node $k, k \in\{1, \ldots, K-1\}$ in the network can be denoted as

$$
\eta_{k}=\frac{\xi\left\|\sum_{i=1}^{K} \mathbf{H}_{k i} \mathbf{V}_{1, i}\right\|^{2}}{\log \left(1+\left|\mathbf{U}_{1, k}^{H} \mathbf{H}_{k k} \mathbf{V}_{1, k}\right|^{2}\right)} .
$$

For $k$ th relay node, the energy that should be shared with AS relay node is

$$
Q_{\mathrm{Req}, k}=\left(v-\frac{1}{\eta_{k}}\right)^{+} .
$$

And $v$ makes $\sum_{k=1}^{K-1} Q_{\text {Req }, k}=Q_{\text {Req. }}$.

However, energy sharing is not always available for the PS relay node since there is a rate requirement at the PS link. For $k$ th link, assume that, when the link rate requirement is satisfied, the saved energy at relay is denoted as $Q_{\text {share, }, k}^{\max }$. Practically, the energy that can be saved in $k$ th rely node is $Q_{\text {share }, k}=\min \left(Q_{\text {share }, k}^{\max }, Q_{\text {Req }, k}\right)$.

Remark 3. If the relay node cannot save any energy when the rate requirement is satisfied, that is, $Q_{\text {share, } k}^{\max }=0$, the AS relay node would not receive any energy from the corresponding relay node.

Finally, $K$ th relay node received energy is $Q_{\text {received }}=$ $\sum_{k=1}^{K-1} Q_{\text {share }, k}$. If the received energy is smaller than the energy requirement $Q_{\text {Req }}$ (the energy cannot afford to forward the information) the AS relay links should be redesigned to maximize their information rate. The algorithm of energy cooperation scheme is concluded as Algorithm 1.

Algorithm 1 (energy cooperation scheme). (1) Calculate AS user's maximum rate $R_{K}^{*}$ and corresponding harvested energy $Q_{K}^{*}$ in Slot 1.

(2) Calculate the energy requirement $Q$ that makes Slot 2 achieve the information rate $R_{K}^{*}$.

(3) For the PS relay nodes satisfy (21a) and (21b), they should totally share energy $Q_{\text {Req }}=Q-Q_{K}^{*}$ to AS relay node.

(4) Calculate the energy that should be saved in $k$ th relay node $Q_{\text {Req }, k}$ by (23).

(5) For $k$ th relay node, calculate the energy that can be saved $Q_{\text {share, } k}^{\max }$ when $k$ th relay node satisfied the rate requirement, and the energy that can be shared with AS relay node is $Q_{\text {share }, k}=\min \left(Q_{\text {share }, k}^{\max }, Q_{\text {Req }, k}\right)$ actually.

(6) Calculate the total received energy $Q_{\text {received }}=$ $\sum_{k=1}^{K-1} Q_{\text {share, }}$ in AS relay.

(7) If $Q_{\text {received }}=Q_{\text {Req }}$, rate of AS link is $R_{K}^{*}$. Else redesign the AS link to maximize their information rate. 


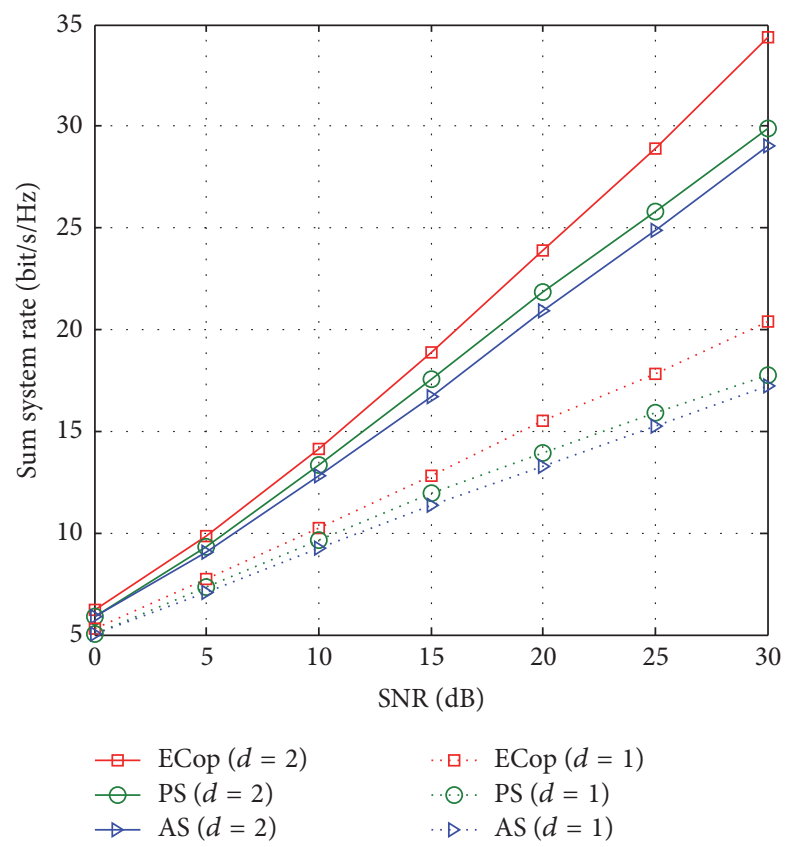

Figure 4: Achievable sum system rate for different strategies.

\section{Simulation Result and Discussions}

In this section, we provide numerical results to compare the performance of investigated schemes.

Firstly consider the achievable rate performances for different transmission strategies at different transfer power level.

For the situation with $d=1$, a three-user IA relay network with $M=N=T=3$ is considered. Assume that, in AS, to meet the IA feasibility condition, $L=2$. For ECop, ID antennas are $L=2$ at the AS node, and receiving antennas at PS relay nodes are $N=3$.

For the situation with $d=2$, assume that $M=6, N=3$, and $T=6$. In the AS scheme, $L=2$. In ECop, ID antennas are $L=2$ at the AS node, and receiving antennas at PS relay nodes are $N=3$. The conventional iterative algorithm is utilized to obtain the solutions for IA transceivers [20].

Figure 4 shows that the PS scheme always has a better performance than AS since the PS scheme makes a more flexible tradeoff between energy and information. Moreover, PS always has a higher DoF condition in any antenna situation. Since the system rate of a relay link depends on the smaller Slot, but both slots happen in the same transmission duration, the performance of PS and AS improves linearly with the increase of transfer power rather than logarithmically.

For ECop, the rate performance outperforms AS and PS. Since the AS node can achieve and maximize information due to no self-energy constraint, hence the ECop scheme achieves a better sum system rate.

In the next experiment, users are distributed randomly in a one-dimensional region. The distance between source node and destination is 200 meters, which can be normalized to 1 . The distance between source node and relay node is denoted as $s$ and for the relay node to destination node denoted as

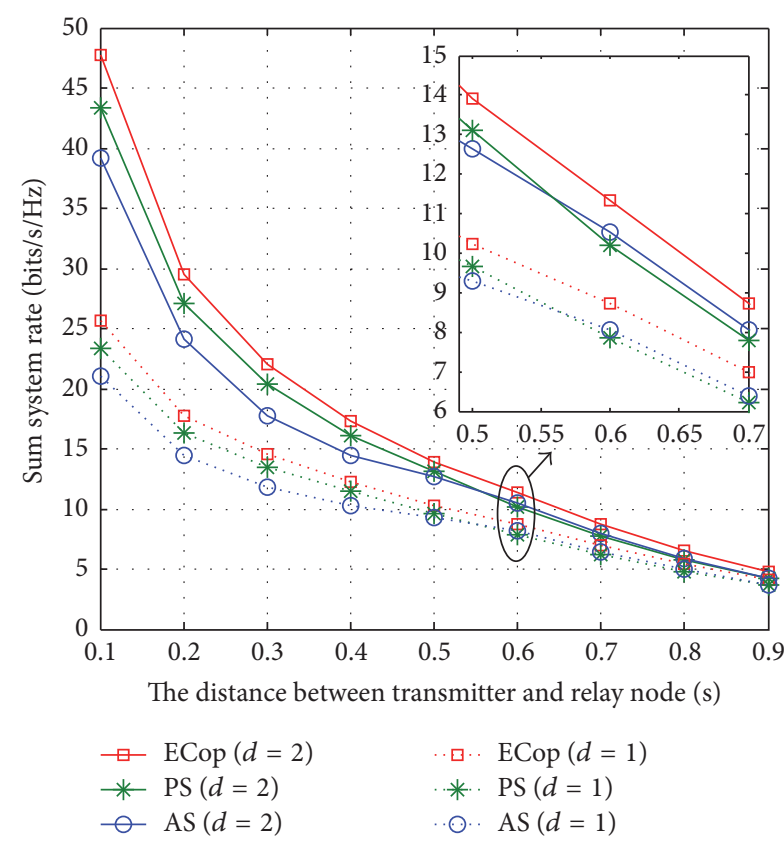

Figure 5: Achievable sum system rate of the IA relay network for different schemes at different relay position.

$1-s$. Suppose the large scale path-loss factor $\phi=3$, and the channel coefficients between relay and users would have additional large scale path-loss $\Omega_{1}=s^{-3}, \Omega_{2}=(1-s)^{-3}$, respectively.

For $d=1$ and $d=2$, the network condition is the same as the previous experiment. It is observed from Figure 5 that, when relay nodes are closer to destination nodes, the AS scheme has a better performance, which indicates that the AS scheme has an advantage on information transmission. This conclusion is somewhat surprising when the UPS was superior to the AS in the conventional MIMO systems by maintaining spatial multiplexing gain at the cost of power loss. It is worth pointing out that AS has a better rate performance at the cost of lower DoF. In summary, AS has a better rate performance with hard DoF condition; on the other hand, UPS has an advantage on the harvested energy with a better DoF condition. For a real communication system, we should choose the receiving scheme according to the actual situations.

Furthermore, it is noted that ECop always has the highest rate which indicates that the proposed scheme can improve the system performance efficiently. ECop can also achieve higher DoF than that of AS.

\section{Conclusions}

In this paper, SWIPT in IA relay networks has been studied. Firstly AS- and PS-based SWIPT is analyzed to maximize the sum-rate of the networks, and a three-step optimization method is proposed. The simulation results show that PS will not always outperform the AS scheme. This conclusion is somewhat surprising when we remember that the UPS always have a bigger R-E region than AS in traditional scenario. 
These results show that these two schemes lead to a new transmission characteristic when interference is taken as the energy source in IA networks.

To combine the advantages of AS and PS, a new ECop scheme is proposed to achieve better performance. Simulation results are presented to show the advantage of the ECop scheme in IA relay networks.

\section{Competing Interests}

The authors declare that there is no conflict of interests regarding the publication of this paper.

\section{Acknowledgments}

This work was partly supported by the National Natural Science Foundation of China (61461029 and 61561032), the Natural Science Foundation of Jiangxi Province (20114ACE00200, 20142BAB217005, and 20142BBE50046), China/Jiangxi Postdoctoral Science Foundation Funded Project (nos. 2013MT541875, 2014MT561879, 2015T80692, and 2014KY046), and Graduate Student Innovation Special Funds of Nanchang University (cx2015138).

\section{References}

[1] L. R. Varshney, "Transporting information and energy simultaneously," in Proceedings of the 2008 IEEE International Symposium on Information Theory (ISIT '08), pp. 1612-1616, Toronto, Canada, July 2008.

[2] P. Grover and A. Sahai, "Shannon meets tesla: wireless information and power transfer," in Proceedings of the IEEE International Symposium on Information Theory (ISIT '10), pp. 2363-2367, IEEE, Austin, Tex, USA, June 2010.

[3] L. Liu, R. Zhang, and K.-C. Chua, "Wireless information transfer with opportunistic energy harvesting," IEEE Transactions on Wireless Communications, vol. 12, no. 1, pp. 288-300, 2013.

[4] R. Zhang and C. K. Ho, "MIMO broadcasting for simultaneous wireless information and power transfer," IEEE Transactions on Wireless Communications, vol. 12, no. 5, pp. 1989-2001, 2013.

[5] Z. Xiang and M. Tao, "Robust beamforming for wireless information and power transmission," IEEE Wireless Communications Letters, vol. 1, no. 4, pp. 372-375, 2012.

[6] H. Ju and R. Zhang, "A novel mode switching scheme utilizing random beamforming for opportunistic energy harvesting," IEEE Transactions on Wireless Communications, vol. 13, no. 4, pp. 2150-2162, 2014.

[7] K. Huang and E. Larsson, "Simultaneous information and power transfer for broadband wireless systems," IEEE Transactions on Signal Processing, vol. 61, no. 23, pp. 5972-5986, 2013.

[8] D. W. K. Ng, E. S. Lo, and R. Schober, "Wireless information and power transfer: energy efficiency optimization in OFDMA systems," IEEE Transactions on Wireless Communications, vol. 12, no. 12, pp. 6352-6370, 2013.

[9] X. Zhou, R. Zhang, and C. K. Ho, "Wireless information and power transfer in multiuser OFDM systems," IEEE Transactions on Wireless Communications, vol. 13, no. 4, pp. 2282-2294, 2014.

[10] J. Xu, L. Liu, and R. Zhang, "Multiuser MISO beamforming for simultaneous wireless information and power transfer," IEEE
Transactions on Signal Processing, vol. 62, no. 18, pp. 4798-4810, 2014.

[11] C. Shen, W.-C. Li, and T.-H. Chang, "Simultaneous information and energy transfer: a Two-user MISO interference channel case," in Proceedings of the 2012 IEEE Global Communications Conference (GLOBECOM '12), pp. 3862-3867, Anaheim, Calif, USA, December 2012.

[12] J. Park and B. Clerckx, "Joint wireless information and energy transfer in a Two-User MIMO interference channel," IEEE Transactions on Wireless Communications, vol. 12, no. 8, pp. 4210-4221, 2013.

[13] K.-B. Huang and E. Larsson, "Simultaneous information and power transfer for broadband wireless systems," IEEE Transactions on Signal Processing, vol. 61, no. 23, pp. 5972-5986, 2013.

[14] B. Wang, J. Zhang, and A. Host-Madsen, "On the capacity of MIMO relay channels," IEEE Transactions on Information Theory, vol. 51, no. 1, pp. 29-43, 2005.

[15] Y.-S. Kim and H. Liu, "Infrastructure relay transmission with cooperative MIMO," IEEE Transactions on Vehicular Technology, vol. 57, no. 4, pp. 2180-2188, 2008.

[16] J. Y. Ryu and W. Choi, "Balanced linear precoding in decodeand-forward based MIMO relay communications," IEEE Transactions on Wireless Communications, vol. 10, no. 7, pp. 23902400, 2011.

[17] B. K. Chalise, Y. D. Zhang, and M. G. Amin, "Energy harvesting in an OSTBC based amplify-and-forward MIMO relay system," in Proceedings of the IEEE International Conference on Acoustics, Speech, and Signal Processing (ICASSP '12), pp. 3201-3204, Kyoto, Japan, March 2012.

[18] A. A. Nasir, X. Zhou, S. Durrani, and R. A. Kennedy, "Relaying protocols for wireless energy harvesting and information processing," IEEE Transactions on Wireless Communications, vol. 12, no. 7, pp. 3622-3636, 2013.

[19] C. Huang, R. Zhang, and S. Cui, "Throughput maximization for the gaussian relay channel with energy harvesting constraints," IEEE Journal on Selected Areas in Communications, vol. 31, no. 8, pp. 1469-1479, 2013.

[20] K. Gomadam, V. R. Cadambe, and S. A. Jafar, "A distributed numerical approach to interference alignment and applications to wireless interference networks," IEEE Transactions on Information Theory, vol. 57, no. 6, pp. 3309-3322, 2011.

[21] C. M. Yetis, T. Gou, S. A. Jafar, and A. H. Kayran, "Feasibility conditionsfor interference alignment," in Proceedings of the IEEE Global Telecommunications Conference (GLOBECOM '09), pp. 1-6, Honolulu, Hawaii, USA, November 2009.

[22] S. A. Jafar, "Interference alignment-a new look at signal dimensions in a communication network," Foundations and Trends in Communications and Information Theory, vol. 7, no. 1, pp. 1-134, 2010.

[23] N. Zhao, F. R. Yu, and V. C. M. Leung, "Wireless energy harvesting in interference alignment networks," IEEE Communications Magazine, vol. 53, no. 6, pp. 72-78, 2015.

[24] X. Li, Y. Sun, F. R. Yu, and N. Zhao, "Antenna selection and power splitting for simultaneous wireless information and power transfer in interference alignment networks," in Proceedings of the IEEE Global Communications Conference (GLOBECOM '14), pp. 2667-2672, Austin, Tex, USA, December 2014. 
[25] S. Boyd and L. Vandenberghe, Convex Optimization, Cambridge University Press, Cambridge, Uk, 2004.

[26] C. M. Yetis, T. Gou, S. A. Jafar, and A. H. Kayran, "On feasibility of interference alignment in MIMO interference networks," IEEE Transactions on Signal Processing, vol. 58, no. 9, pp. 47714782, 2010. 

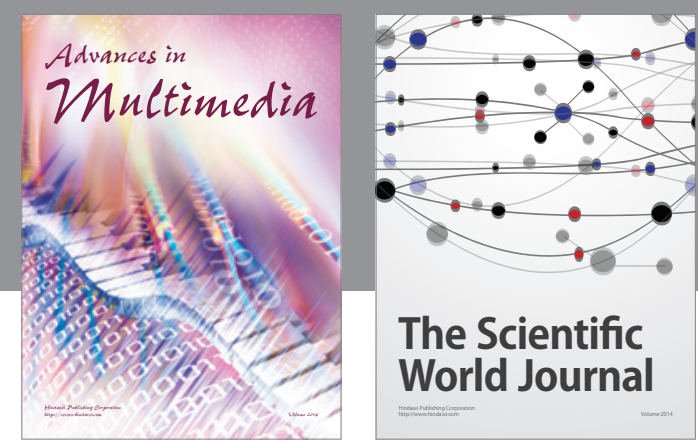

The Scientific World Journal
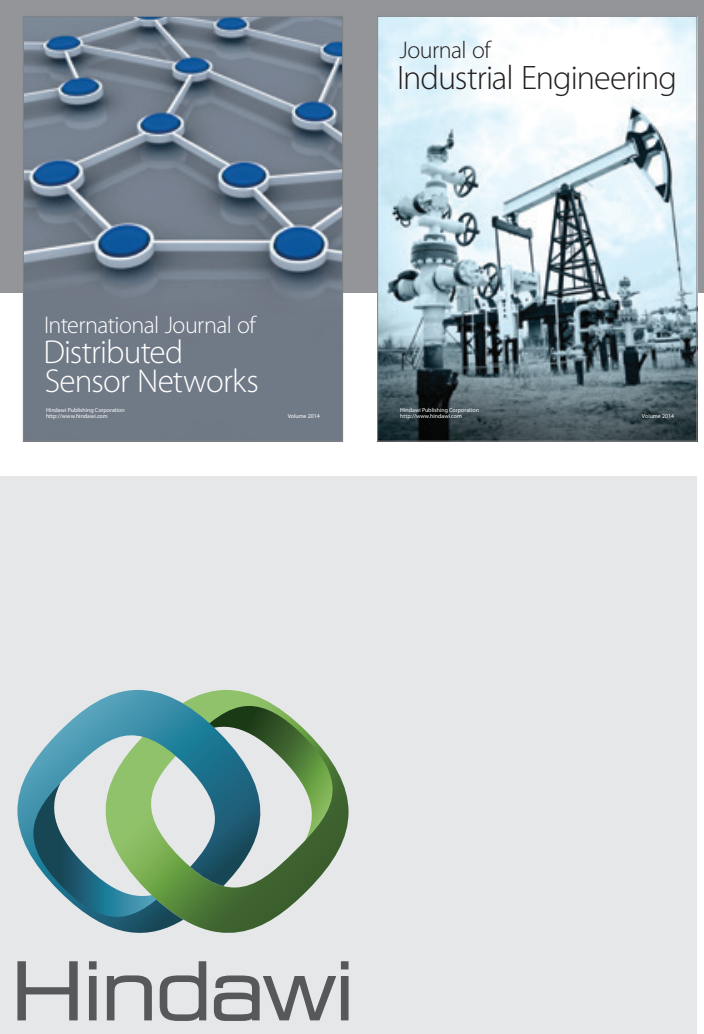

Submit your manuscripts at

http://www.hindawi.com

\section{Computer Networks} and Communications
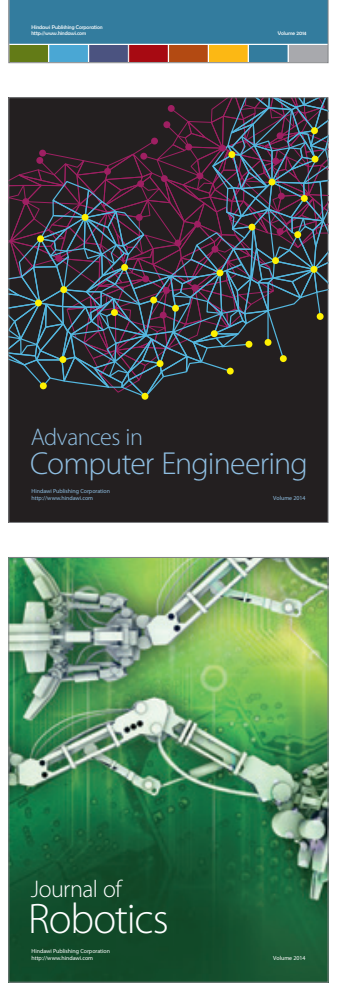
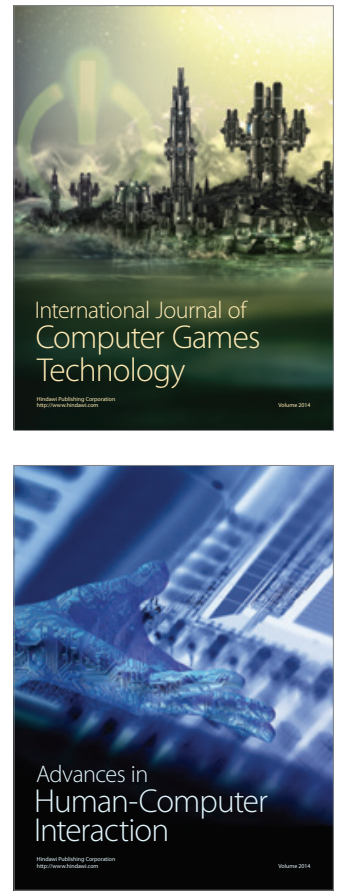
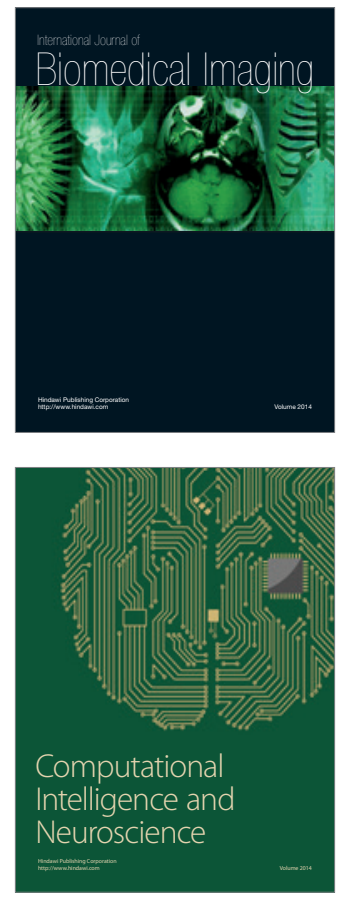
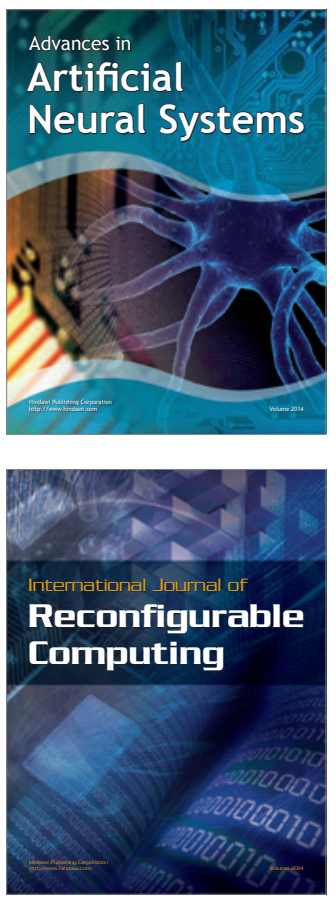
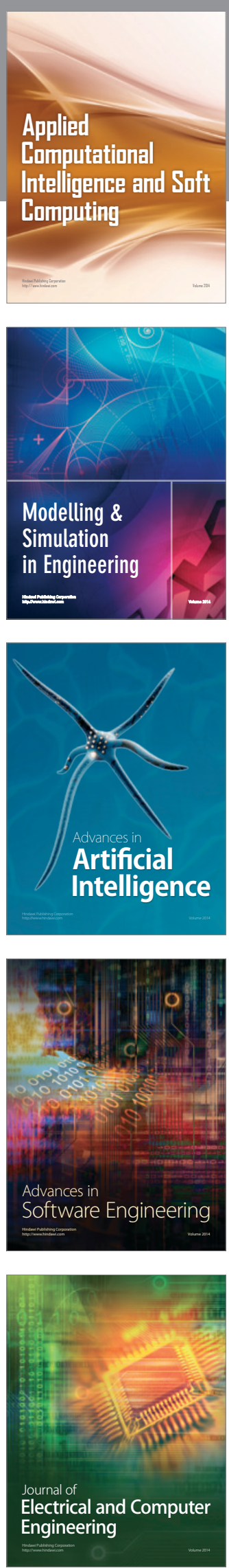